\title{
Application of different lipid surfaces to monitor protein-membrane interactions by surface plasmon resonance spectroscopy
}

\author{
Torsten Fischer, ${ }^{\mathrm{a}, 1}$ Ivan I. Senin ${ }^{\mathrm{b}}$, Pavel P. Philippov ${ }^{\mathrm{b}}$ and Karl-Wilhelm Koch ${ }^{\mathrm{a}, *}$ \\ ${ }^{a}$ Institut für Biologische Informationsverarbeitung 1, Forschungszentrum Jülich, D-52425 Jülich, \\ Germany \\ ${ }^{\mathrm{b}}$ A.N. Belozersky Institute of Physico-Chemical Biology, Moscow State University, 119992 Moscow, \\ Russia
}

\begin{abstract}
Planar lipid bilayers on sensor chip surfaces have become useful tools to study membrane bound processes by surface plasmon resonance spectroscopy. We immobilized phospholipids on sensor chips by different approaches. First, a selfassembled monolayer of octadecylmercaptan was formed on a blank gold surface and subsequent addition of phospholipids led to formation of a heterobilayer. Second, a self-assembled monolayer of mercaptoundecanoic acid was formed on a gold surface, the carboxy groups of mercaptoundecanoic acid were activated and covalently linked to phosphatidylethanolamine. Addition of phospholipids then led to a bilayer with phosphatidylethanolamine as the lower leaflet. Third, a hydrophobic sensor chip (L1, BIAcore) was used as a binding matrix for phospholipids. These lipid surfaces were tested, whether they are suitable to study protein-membrane interactions. As biological test system we used the $\mathrm{Ca}^{2+}$-myristoyl-switch of the neuronal $\mathrm{Ca}^{2+}$ binding protein recoverin. All three surfaces were sufficiently stable to monitor the $\mathrm{Ca}^{2+}$-dependent binding of recoverin to membranes.
\end{abstract}

\section{Abbreviations}

ROS, bovine rod outer segments; PMSF, phenylmethyl-sulfonylfluoride; SPR, surface plasmon resonance spectroscopy; PE, phosphatidylethanolamine; PC, phosphatidylcholine; PS, phosphatidylserine; SAM, self-assembled monolayer; NHS, N-hydroxysuccinimid; EDC, N-ethyl-N'-[(dimethylamino)propyl]carbodiimide; ODM, octadecylmercaptan; MUA, mercaptoundecanoic acid.

\section{Introduction}

Evanescent wave biosensors have attained wide interest in the last years. They employ surface plasmon resonance (SPR) spectroscopy to study the interaction of biological macromolecules, in particular the association and dissociation of proteins and peptides [11,20,21]. The investigation of protein-protein interactions, that involve integral membrane proteins or membrane associated proteins is of special importance for fields like signal transduction and nerve signalling. Since biosensor technology requires the immobilization of one binding partner on the chip surface, the immobilization of membrane proteins is

\footnotetext{
${ }^{1}$ Current address: Institute of Genetic Medicine and Neurogenetic Institute, Departament of Biochemistry and Molecular Biology, UCLA, CA 90033, USA.

${ }^{*}$ Corresponding author. Tel.: +49 246161 3255; Fax: +49 2461 614216; E-mail: k.w.koch@fz-juelich.de.
} 
a challenging problem. Since membrane proteins need a hydrophobic environment, this approach then requires the immobilization of phospholipids and the incorporation of the membrane protein into the bilayer. In addition, the immobilized lipid bilayer must be of sufficient stability and must withstand regenerations pulses to disrupt a preformed protein-protein complex. The bilayer must further maintain its structure, as any significant loss of lipids during the recording of a sensorgram would cause a drift in the baseline and therefore will complicate the kinetic analysis. Loss of lipids can also lead to gaps in the bilayer structure.

Several approaches have been used so far to tether lipids on solid supports (sensor chip surfaces) including direct deposition on the surface [19], fusion on a hydrophobic self assembled monolayer (SAM) of alkanethiols [5,18], binding of biotin-labelled liposomes to streptavidin-coated sensor chips $[4,16,17]$ and binding to patterned SAM [3,9]. We tested three different methods for assembling of lipids on solid supports. Bilayers were used to study the $\mathrm{Ca}^{2+}$-dependent membrane association of recoverin [6,15], a myristoylated $\mathrm{Ca}^{2+}$-binding protein, that undergoes a $\mathrm{Ca}^{2+}$-induced structural change with the exposition of its covalently attached myristoyl group $[2,7,23]$. Since this process represents a reversible binding event to membranes and since it is controlled by a change in free $\mathrm{Ca}^{2+}$-concentration, it is ideally suited to test the applicability of different lipid layers on solid supports.

\section{Experimental procedures}

\subsection{Preparation of lipid vesicles from rod outer segment membranes}

Bovine rod outer segments (ROS) and washed ROS membranes were prepared as previously described [14]. ROS membranes were solubilized in $2 \%(\mathrm{w} / \mathrm{v})$ octylglucoside, $20 \mathrm{mM} \mathrm{HEPES} / \mathrm{KOH} \mathrm{pH}$ 7.4, $100 \mathrm{mM} \mathrm{KCl}, 1 \mathrm{mM}$ DTT and $0.1 \mathrm{mM}$ PMSF and centrifuged for $30 \mathrm{~min}$ at 45,000 rpm (TLA-45 rotor, Beckman). The supernatant was dilaysed against SPR running buffer (10 mM HEPES/KOH pH 7.4, $150 \mathrm{mM} \mathrm{NaCl}, 20 \mathrm{mM} \mathrm{MgCl} 2,2 \mathrm{mM} \mathrm{CaCl}_{2}$ ).

\subsection{Preparation of liposomes}

Lipids were dissolved in chloroform $(10 \mathrm{mg} / \mathrm{ml})$ and dried down by vacuum in a speed vac concentrator at room temperature. The lipid pellet was resuspended in $10 \mathrm{mM} \mathrm{HEPES} / \mathrm{KOH} \mathrm{pH} \mathrm{7.4,150} \mathrm{mM} \mathrm{NaCl}$ and sonified for $20 \mathrm{~s}$ (Branson B12; $100 \mathrm{~W}$ ). Large unilammelar vesicles (liposomes) were produced using the extrusion technique. The suspension was soaked for 15-20 min and extruded through polycarbonate filter with a pore diameter of $1 \mu \mathrm{m}$ (first step) and $0.4 \mu \mathrm{m}$ (second step).

Liposomes corresponding to the lipid composition in bovine ROS membranes were prepared as described [16] with slight modification. A mixture of $4 \mathrm{mg}$ lipids (40\%w/w PE, $40 \% \mathrm{w} / \mathrm{w}$ PC, $15 \%$ w/w PS; $5 \% \mathrm{w} / \mathrm{w}$ cholesterol in $\mathrm{CHCl}_{3}$ was dried down by vacuum in a speed vac concentrator. The sample was resuspended in $2 \mathrm{ml}$ of degassed buffer (20 mM Hepes pH 7.5, $150 \mathrm{mM} \mathrm{KCl}, 3 \mathrm{mM}$ EGTA) and sonified for $2 \times 15$ min (Branson B12; cup; $100 \mathrm{~W}$ ). Prior to use large unilammelar vesicles were produced as described above.

\subsection{Generation of SAM-chip surfaces}

We recycled used CM5 sensor chips (BIAcore) for the generation of self assembled monolayers (SAM) surfaces [18]. The dextran layer on commercially available CM5 chips is attached to the glass/gold 
support via gold-thiol interaction. For our purpose, this gold-thiol bond was oxidized by strong UVlight for 1-2 hours. Alternatively, the dextran layer was removed by mild treatment with dextranase (56 units/mg) in $10 \mathrm{mM}$ Mes pH 6.15, $150 \mathrm{mM} \mathrm{NaCl}, 10 \mathrm{mM} \mathrm{MgCl} 2$. Chips were incubated in a total volume of $100 \mu \mathrm{l}$ at $37^{\circ} \mathrm{C}$ overnight. Afterwards, chips were washed with $\mathrm{H}_{2} \mathrm{O}_{\text {bidest }}$.

Alkanethiols as octadecylmercaptan or mercaptoundecanoic acid were dissolved in ethanol in a concentration of $10 \mathrm{mM}$ and flushed over a gold surface of a regenerated CM5 sensor chip to form spontaneously a self-assembled monolayer. Details of the procedure were described elsewhere [10].

\subsection{Surface plasmon resonance measurements}

Formation of SAM, immobilization of lipids and binding of recoverin to liposomes and ROS membranes was monitored by SPR spectroscopy. Details of the instrumental setup and examples of different SPR applications can be found elsewhere [10,11,13,16,20,21].

\subsection{Immobilization of lipids}

Liposomes were immobilized on a sensor chip by three different strategies. First a hydrophobic SAM consisting of octadecylmercaptan was formed on the blank gold surface and lipids or lipid mixtures were flushed over the surface in SPR running buffer. Second, a SAM of mercaptoundecanoic acid was formed on the gold surface. The carboxy groups were activated by $50 \mathrm{mM}$ N-hydroxysuccinimid (NHS) and $200 \mathrm{mM}$ N-ethyl-N'-[(dimethylamino)propyl]carbodiimide (EDC) for 7 minutes at a flow rate of $5 \mu \mathrm{l} / \mathrm{min}$ (injection volume $35 \mu \mathrm{l}$ ). Phosphatidylethanolamine (PE) was dissolved in $50 \mathrm{mM} \mathrm{Na}$-formiate buffer $\mathrm{pH} 4.0(0.5 \mathrm{mg} / \mathrm{ml})$ and applied into the flow cell. The free amino groups of PE reacted with the activated carboxy groups. Afterwards, a solution of $1 \mathrm{M}$ ethanolamine $\mathrm{pH} 8.5$ was injected for 8 minutes to deactivate excessive reactive groups. Lipids were applied to form a bilayer. Third, lipid solutions were flushed over a hydrophobic sensor chip (pioneer L1, BIAcore), where they formed spontaneously a bilayer. Lipid layers settled, when running buffer without lipids was flowing through the flow cell. Final adjustment of the heterobilayer was achieved by short pulses of $50 \mathrm{mM} \mathrm{NaOH}$ and $10 \mathrm{mM}$ glycine/HCl $\mathrm{pH}$ 2.5. Nonspecific binding was tested by application of calmodulin or protein G. Interaction of recoverin with immobilized liposomes or ROS vesicles was tested by applying 5-30 $\mu \mathrm{M}$ protein in running buffer at saturating $\left[\mathrm{Ca}^{2+}\right]$ and $20 \mathrm{mM} \mathrm{MgCl} 2$ at $\mathrm{pH}$ 7.1. The flow rate was $5 \mu \mathrm{l} / \mathrm{min}$. Resonance signals at equilbrium were determined as described. Data evaluation was performed with the software BIAevaluation 3.1.

\subsection{Purification of recoverin}

Native recoverin was purified from frozen bovine retinae as described [16]. Myristoylated wildtype recoverin was coexpressed in E. coli with yeast $\mathrm{N}$-myristoyl-transferase and purified as described [1].

\section{Results and discussion}

\subsection{Formation of different lipid surfaces}

SPR spectroscopy was applied to study the $\mathrm{Ca}^{2+}$-dependent association of the neuronal $\mathrm{Ca}^{2+}$-binding protein recoverin to immobilized phospholipid membranes. We investigated the formation and stability 


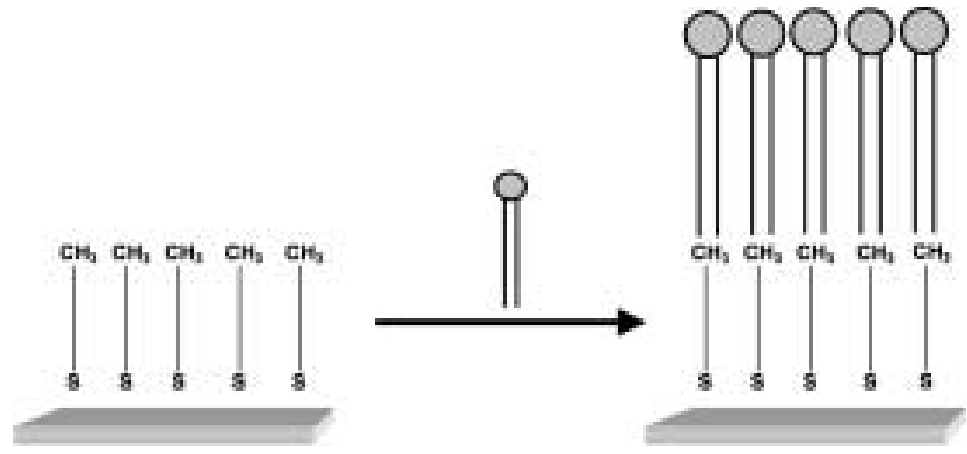

Fig. 1. Formation of a heterobilayer on a self-assembled monolayer of octadecylmercaptan.

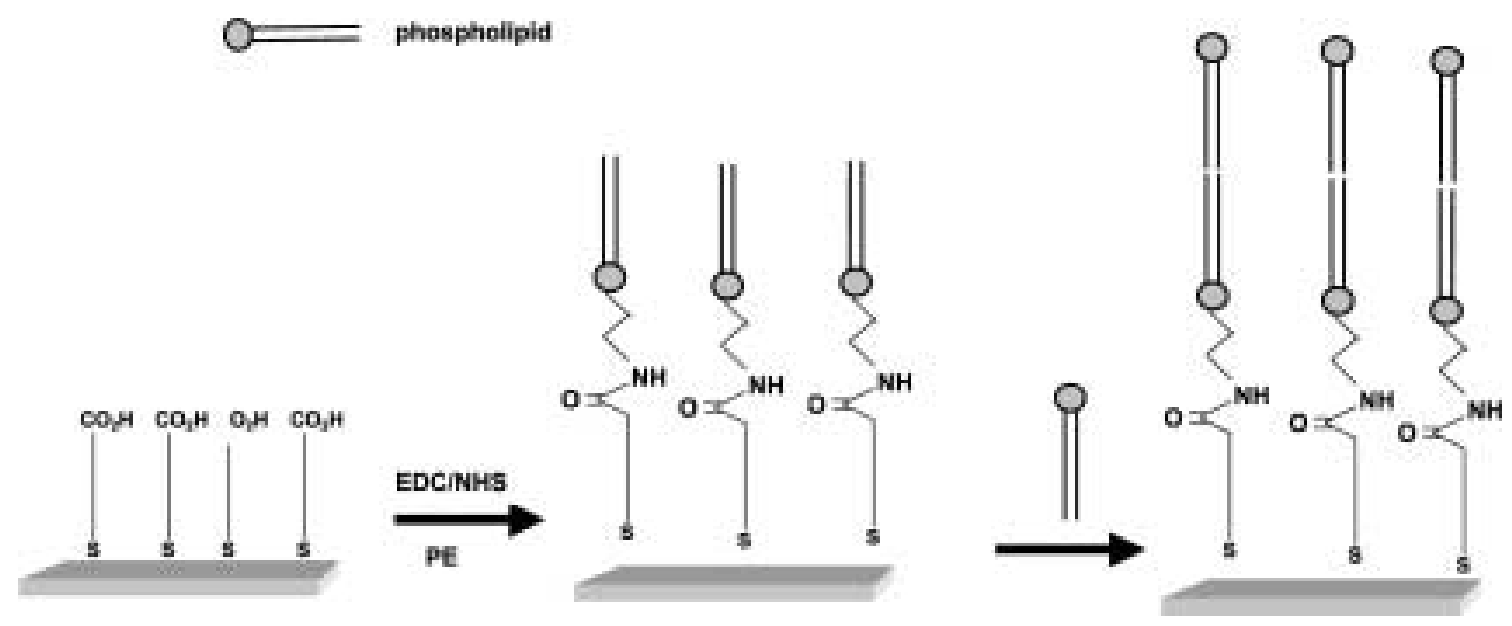

(a)

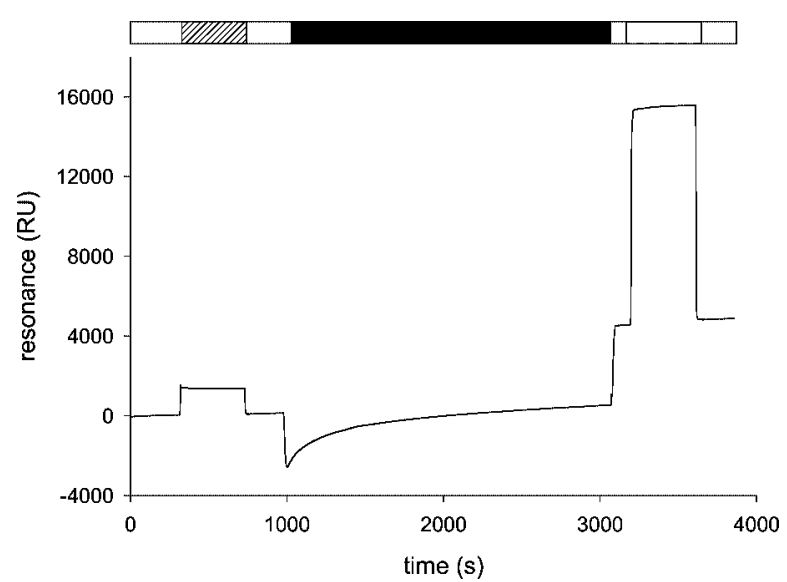

(b)

Fig. 2. (a) Covalent coupling of phosphatidylethanolamine (PE) to mercaptoundecanoic acid and subsequent formation of a phospholipid bilayer. (b) Sensorgram shoowing the covalent coupling of phosphatidylethanolamine (PE) to activated mercaptoundecanoic acid. 


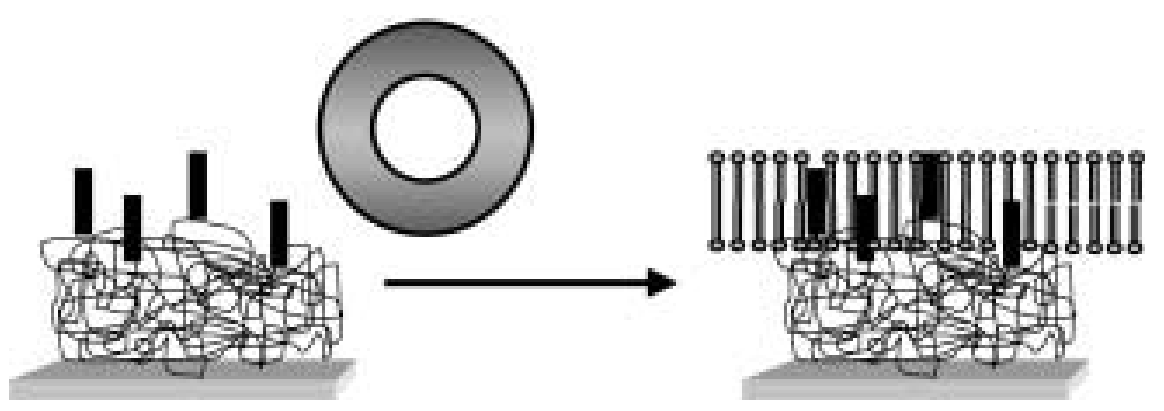

(a)



(b)

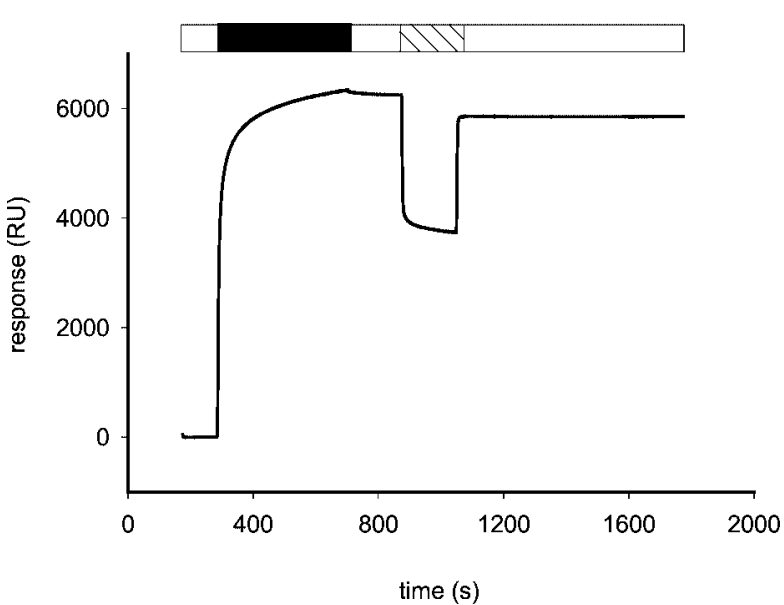

(c)

Fig. 3. (a) Immobilization of liposomes on a sensor chip coated with hydrophobic anchors (L1). (b) Sensorgram showing immobilization of liposomes on a L1-chip by repeated injections in the presence of divalent cations. (c) Sensorgram showing immobilization of liposomes on a L1-chip in the absence of divalent cations.

of different lipid layers on sensor surfaces. Three different strategies were tested to immobilize lipids on sensor chip surfaces, they are explained schematically in Figs 1-3.

First, a commercially CM5 sensor chip (BIAcore) that had been used in previous experiments was regenerated as described $[10,18]$. This process led to a blank gold surface. A solution of octadecylmercaptan (ODM) that was layered on the gold surface formed a self-assembled monolayer of ODM (Fig. 1). Phospholipids were added to form a heterobilayer by association of the fatty acyl chains to the hydrophobic alkane chains of the ODM layer. In this heterobilayer, the polar head groups extend into the buffer flow reservoir (flow cell of the BIAcore system).

Second, this approach also starts with the regeneration of used CM5 sensor chips, but the self assembled monolayer on the surface is formed by mercaptoundecanoic acid (MUA) (Fig. 2(a)). The introduction of carboxy groups allows to modify this monolayer by chemical procedures. We coupled 
phosphatidylethanolamine (PE) directly to the MUA layer by amide formation (Fig. 2(a)). By this step we immobilized a phospholipid layer with an extension of its hydrophobic acyl chains into the flow cell (PE-MUA surface). Application of a lipid mixture then forms a bilayer by selfassembling (PE-MUA bilayer). A typical sensorgram that shows the different steps during the coating of the surface is shown in Fig. 2(b). The carboxy groups on the MUA layer were activated by EDC/NHS (hatched bar), phosphatidylethanolamine was supplied and reacted with the activated surface (black bar). Activated carboxy groups that had not reacted were deactivated by ethanolamine (gray bar). Lipid vesicles were injected into the flow cell and flushed over the PE-MUA surface to form the bilayer (not shown).

Third, we used a commercially available sensor chip (L1, BIAcore) that consists of a carboxy-dextran matrix coated with small hydrophobic anchor molecules [12]. This surface allows the formation of planar phospholipid bilayers within the hydrophobic anchors (Fig. 3(a)). The sensorgram in Fig. 3(b) and (c) show the application of a lipid mix into the flow cell (black bars) and the adjustment of the bilayer on the surface after a pulse of $50 \mathrm{mM} \mathrm{NaOH}$ (hatched bars). Divalent cations as $\mathrm{Mg}^{2+}$ and $\mathrm{Ca}^{2+}$ caused a slower assembling of phospholipids on the chip surface (Fig. 3(b)). The presence of the chelator EGTA (no addition of $\mathrm{Mg}^{2+}$ and $\mathrm{Ca}^{2+}$ ) led to an accelerated immobilization of phospholipids, i.e., saturation of the surface was achieved in shorter time (Fig. 3(c)). The stability of the bilayer was similar in both cases.

\subsection{Stability of lipid layers}

The geometry of the ODM-heterobilayer (Fig. 1(a)) is best suited to test, whether the stability of the lipid layer depends on the lipid composition. The selfassembled alkane layer forms a simple and homogeneous platform, on which the composition of the lipid layer can be varied. The stability was tested by measuring the change in resonance units at specific time intervals and is seen for several phospholipids and phospholipid mixtures in Fig. 4. The most stable heterobilayer was observed with phosphatidylserine (PS) and dipalmitoyl-phosphaditylethanolamine (PE-Dip). Phosphaditylcholine (PC) and dimyristoyl-phosphatidylcholine (PC-Dim) were of intermediate stability. The least stable layers were

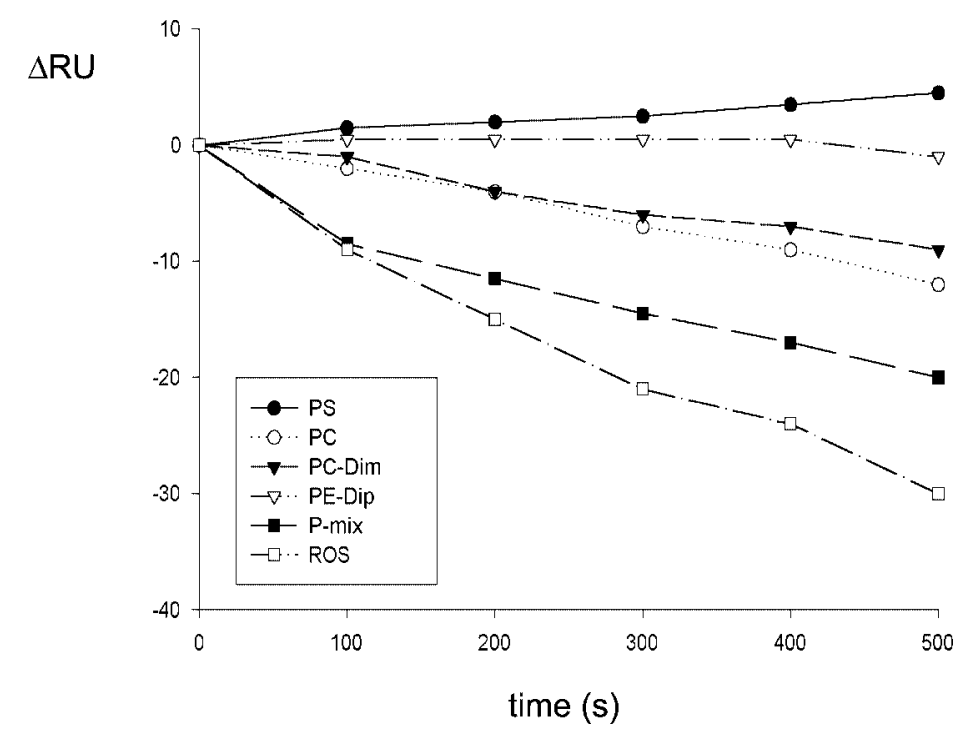

Fig. 4. Stability of lipid layers that were tested on an octadecylmercaptan heterobilayer. 
formed by the lipid mixture P-mix, which corresponds to the lipid composition of retinal rod outer segments [22] and ROS, which were native rod outer segment membranes. Instability of ROS membranes was mainly due to the presence of unsaturated fatty acids in these membranes [22], that cause a high fluidity and a less ordered leaflet structure. However, all surfaces lost not more than $1 \%$ of the total amount of immobilized lipids within one hour. Thus, they are suitable for recording a sensorgram in a time frame of 20-30 minutes.

\section{3. $\mathrm{Ca}^{2+}$-myristoyl switch of recoverin as biological test system for immobilized lipids}

As biological test system we used the $\mathrm{Ca}^{2+}$-dependent association of the neuronal $\mathrm{Ca}^{2+}$-binding protein recoverin to immobilized lipid layers. Recoverin is a $\mathrm{Ca}^{2+}$-sensor in photoreceptor cells of the vertebrate retina and heterogenously acylated at its $\mathrm{N}$-terminus [7]. The main acyl group is a myristoyl-group that is buried inside a hydrophobic pocket of recoverin when the free $\left[\mathrm{Ca}^{2+}\right]$ is low (below $100 \mathrm{nM}$ ). Increase of $\left[\mathrm{Ca}^{2+}\right]$ triggers the exposition of the myristoyl group into the bulk medium and in consequence the protein is anchored to membranes [2,7,23]. This $\mathrm{Ca}^{2+}$-myristoyl-switch is thought to be a critical trigger mechanism during illumination of a photoreceptor cell. Dynamics of this $\mathrm{Ca}^{2+}$-myristoyl switch have been studied before using SPR spectroscopy [16].

We applied this test system to compare different bilayers (Figs 1-3). Recoverin (5-10 $\mu \mathrm{M})$ was supplied in a SPR running buffer that contained saturating $\mathrm{Ca}^{2+}(2 \mathrm{mM})$. Injection of recoverin into the flow cell (black bar in Fig. 5) caused an increase in RU, which indicates association of recoverin with immobilized lipids. Dissociation from the surface is achieved by flushing the cell with running buffer (no recoverin, open bar in Fig. 5). Finally, a short pulse of the $\mathrm{Ca}^{2+}$-chelator EGTA was applied (hatched bar in Fig. 5) to trigger complete dissociation of recoverin from the bilayer. Seonsorgrams were recorded on an ODM-heterobilayer (trace 1) and on the PE-MUA bilayer (trace 2). Although both sensorgrams were similar, they displayed some characteristic differences. For example, association of recoverin to the PE-MUA bilayer reached almost a plateau during the time course of the association phase (trace 2), but in the case of the ODM-heterobilayer, the signal still increased after the end of recoverin injection

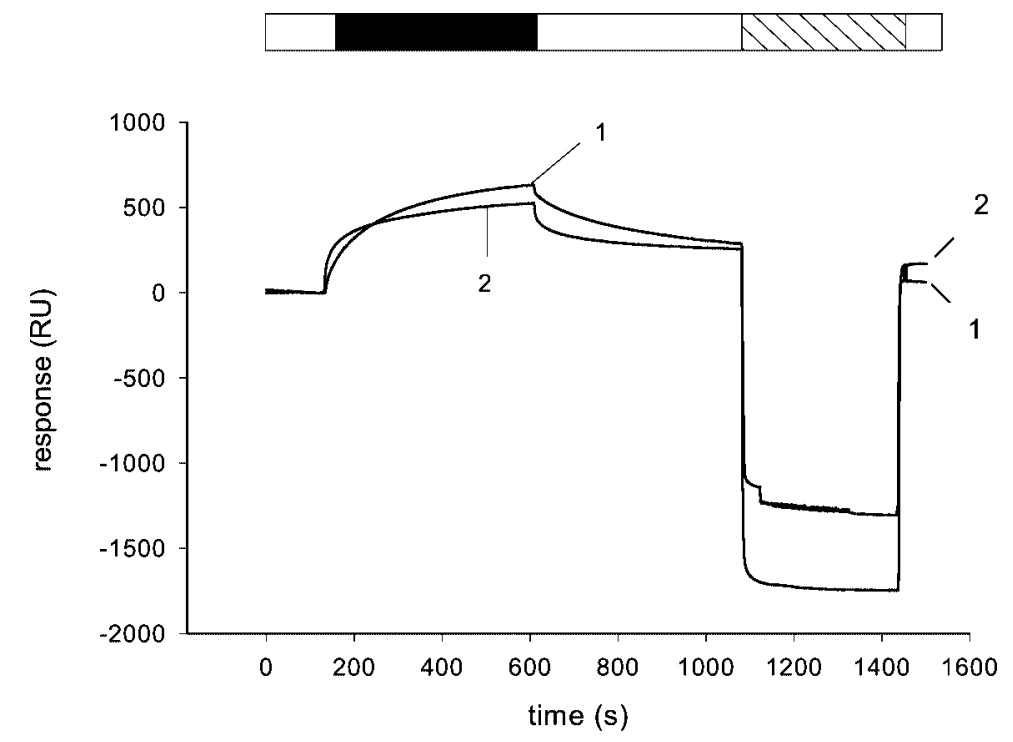

Fig. 5. $\mathrm{Ca}^{2+}$-dependent binding of recoverin to immobilized lipids (ODM-heterobilayer, trace 1 and PE-MUA bilayer, trace 2). 


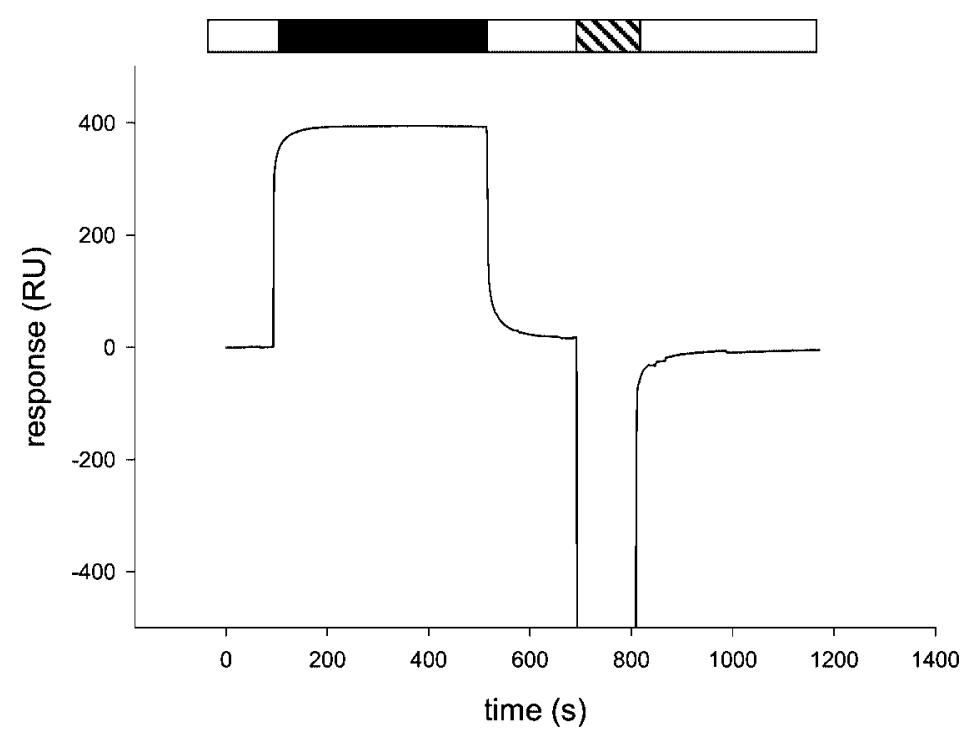

Fig. 6. $\mathrm{Ca}^{2+}$-dependent binding of recoverin to immobilized lipids (hydrophobic anchor chip L1).

(trace 1). This indicated, that the association process might have been limited by mass transfer in the case of the ODM-heterobilayer. The dissociation phases of both sensorgrams also differed, trace 2 reached a plateau and application of EGTA did not cause a complete dissociation of recoverin from the PE-MUA bilayer. In contrast, sensorgram 1 did not form a plateau during the dissociation phase and the baseline was reached after regeneration with EGTA. It seems, that recoverin is trapped on the PE-MUA bilayer, because it did not dissociate completely. We suggest, that the PE-MUA bilayer is less homogeneous. The covalent binding of PE to the EDC/NHS activated carboxy surface probably did not occur with equal spacing, thus resulting in a less ordered PE leaflet and in consequence a less ordered bilayer with gaps. Recoverin might have been trapped in these gaps.

A rather stable phospholipid bilayer was formed on a L1 chip. When we tested the binding of recoverin to immobilized phospholipids, the signal reached an apparent equilibrium during application of $30 \mu \mathrm{M}$ recoverin (black bar in Fig. 6). The dissociation phase did not form a plateau above the baseline and recoverin was completely removed from the surface by regeneration with EGTA. It appeared that no rebinding to the surface during dissociation occurred (formation of a plateau) and that recoverin is not trapped in possible gaps of the lipid layer (s. above).

\section{Conclusion}

Our results showed that all tested immobilized lipid layers are of sufficient stability to allow recording of protein-membrane interactions by SPR spectroscopy. However, kinetics of binding and dissociation of recoverin were not equal and depended on the surface modification used to immobilize the lipids.

\section{Acknowledgements}

This work was supported by a grant (KO948/5-2/5-3) from the Deutche Forschungsgemeinschaft (K.W.K) and in part by grants from the Ludwig Institute for Cancer Research (P.P.Ph) and Russian Foundation for Basic Research N00-04-48332 (P.P.Ph). 


\section{References}

[1] A.M. Alekseev, S.V. Shulga-Morskoy, D.V. Zinchenko, S.A. Shulga-Morskaya, D.V. Suchkov, S.A. Vaganova, I.I. Senin, A.A. Zargarov, V.M. Lipkin, M. Akhtar and P.P. Philippov, Obtaining and characterization of EF-hand mutants of recoverin, FEBS Lett. 440 (1998), 116-118.

[2] J.B. Ames, T. Tanaka, L. Stryer and M. Ikura, Portrait of a myristoyl switch protein, Curr. Opin. Struct. Biol. 6 (1996), 432-438.

[3] C. Bieri, O.P. Ernst, S. Heyse, K.P. Hofmann and H. Vogel, Micropatterned immobilization of a G-protein-coupled receptor and direct detection of G-protein activation, Nature Biotechnol. 17 (1999), 1105-1108.

[4] M.A. Cooper, A. Hansson, S. Löfås and D.H. Williams, A vesicle capture sensor chip for kinetic analysis of interactions with membrane-bond receptors, Analyt. Biochem. 277 (2000), 196-205.

[5] M.A. Cooper, A.C. Try, J. Carroll, D.J. Ellar and D.H. Williams, Surface plasmon resonance analysis at a supported lipid monolayer, Biochim. Biophys. Acta 1373 (1998), 101-111.

[6] A.M. Dizhoor, C.-K. Chen, E. Olshevskaya, V.V. Sinelnikova, P. Phillipov and J.B. Hurley, Role of the acylated amino terminus of recoverin in $\mathrm{Ca}^{2+}$-dependent membrane interaction, Science 259 (1993), 829-832.

[7] A.M. Dizhoor, L.H. Ericsson, R.S. Johnson, S. Kumar, E. Olshevskaya, S. Zozulya, T.A. Neubert, L. Stryer, J.B. Hurley and K.A. Walsh, The $\mathrm{NH}_{2}$ terminus of retinal recoverin is acylated by a small family of fatty acids, J. Biolog. Chem. 267 (1992), 16033-16036.

[8] A.M. Dizhoor, S. Ray, S. Kumar, G. Niemi, M. Spencer, D. Brolley, K.A. Walsh, P.P. Philipov, J.B. Hurley and L. Stryer, Recoverin: A calcium sensitive activator of retinal rod guanylate cyclase, Science 251 (1991), 915-918.

[9] O.P. Ernst, C. Bieri, H. Vogel and K.P. Hofmann, Intrinsic biophysical monitors of transducin activation: Fluorescence, UV-visible spectroscopy, light scattering, and evanescent field techniques, Methods Enzymol. 315 (2000), 471-489.

[10] T. Fischer, M. Beyermann and K.-W. Koch, Application of different surface plasmon resonance biosensory chips to monitor the interaction of the CaM-binding site of nitric oxide synthase I and calmodulin, Biochem. Biophys. Res. Comm. 285 (2001), 463-469.

[11] U. Jönsson, L. Fägerstam, B. Ivarsson, B. Johnsson, R. Karlsson, K. Lundh, S. Löfås, B. Persson, H. Roos, I. Rönnberg, S. Sjölander, E. Stenberg, R. Ståhlberg, C. Urbaniczky, H. Östlin and M. Malmqvist, Real-time biospecific interaction analysis using surface plasmon resonance and a sensor chip technology, BioFeature 11 (1991), 620-627.

[12] O.P. Karlsson and S. Löfås, Flow-mediated on-surface reconstitution of G-protein-coupled receptors for applications in surface plasmon resonance biosensors, Analyt. Biochem. 300 (2002), 132-138.

[13] K.-W. Koch, Identification and characterization of calmodulin binding sites in cGMP-gated channel using surface plasmon resonance spectroscopy, Methods Enzymol. 315 (2000), 785-797.

[14] K.-W. Koch, H.-G. Lambrecht, M. Haberecht, D. Redburn and H.H.H.W. Schmidt, Functional coupling of a $\mathrm{Ca}^{2+} /$ calmodulin-dependent nitric oxide synthase and a soluble guanylyl cyclase in vertebrate photoreceptor cells, EMBO J. 13 (1994), 3312-3320.

[15] H.-G. Lambrecht and K.-W. Koch, A $26 \mathrm{kd}$ calcium binding protein from bovine rod outer segments as modulator of photoreceptor guanylate cyclase, EMBO J. 10 (1991), 793-798.

[16] C. Lange and K.-W. Koch, Calcium-dependent binding of recoverin to membranes monitored by surface plasmon resonance spectroscopy in real time, Biochemistry 36 (1997), 12 019-12 026.

[17] L. Masson, A. Mazza and R. Brousseau, Stable immobilization of lipid vesicles forkinetic studies using surface plasmon resonance, Analyt. Biochem. 218 (1994), 405-412.

[18] A.L. Plant, M. Brigham-Burke, E.C. Petrella and D.J. O'Shannessy, Phospholipid/alkanethiol bilayers for cell surface receptor studies by surface plasmon resonance, Analyt. Biochem. 226 (1995), 342-348.

[19] G. Puu and I. Gustafson, Planar lipid bilayers on solid supports from liposomes - factors of importance for kinetics and stability, Biochim. Biophys. Acta 1327 (1997), 149-161.

[20] R.L. Rich and D.G. Myszka, Survey of the year 2000 commercial optical biosensor literature, J. Molec. Recogn. 14 (2001), 273-294.

[21] P. Schuck, Use of surface plasmon resonance to probe the equilibrium and dynamic aspects of interactions between biological macromolecules, Ann. Rev. Biophys. Biomolec. Struct. 26 (1997), 541-566.

[22] R.D. Wiegand and R.E. Anderson, Determination of molecular species of rod outer segment phospholipids, Methods Enzymol. 81 (1982), 297-304

[23] S. Zozulya and L. Stryer, Calcium-myristoyl protein switch, Proc. Nat. Acad. Sci. USA 89 (1992), 11 569-11 573. 


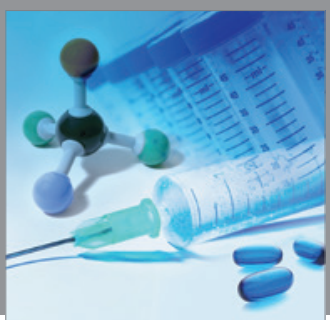

International Journal of

Medicinal Chemistry

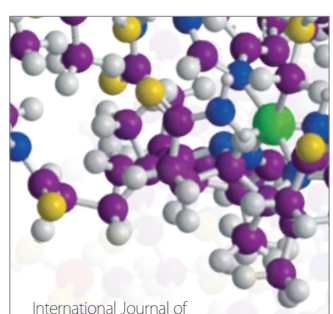

Carbohydrate Chemistry



The Scientific World Journal
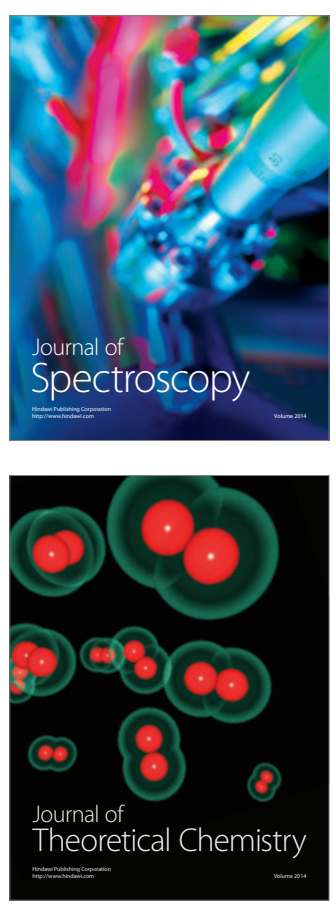
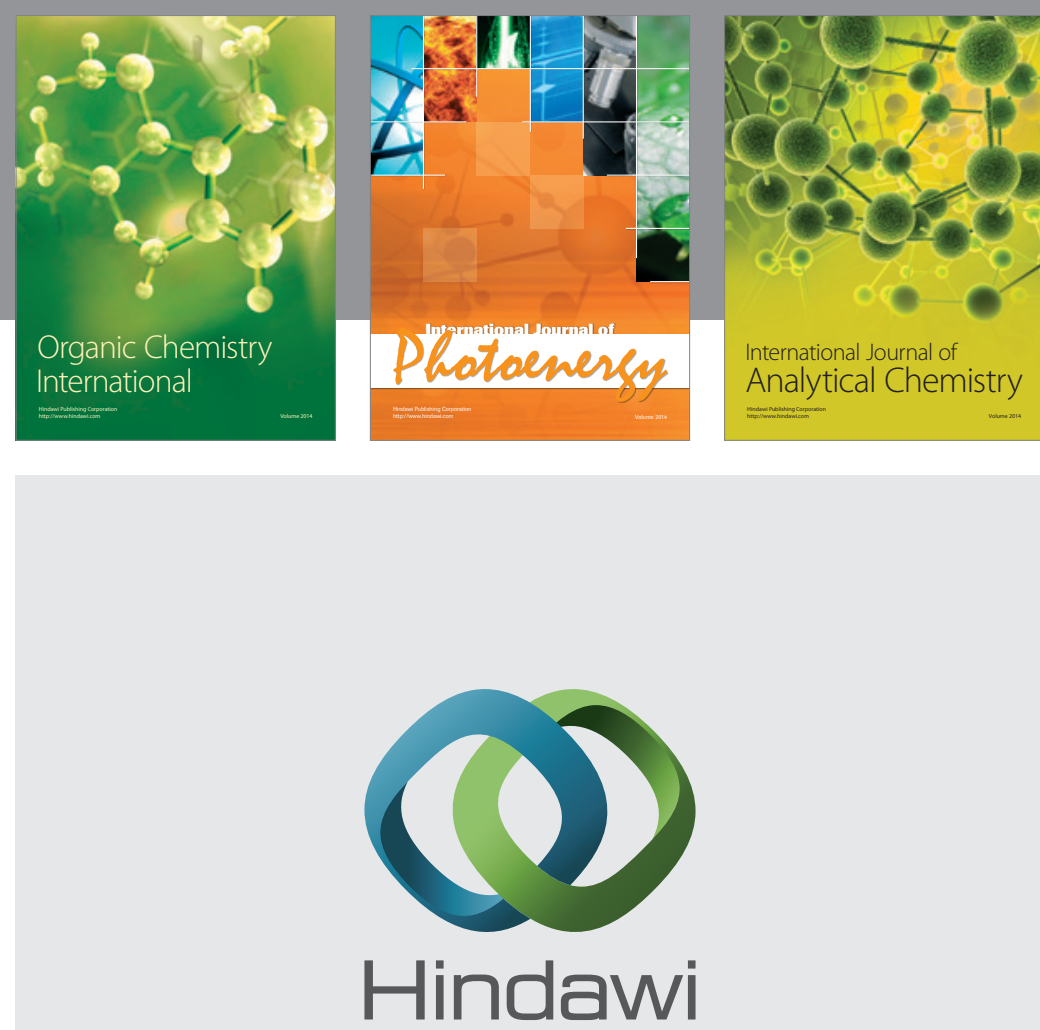

Submit your manuscripts at

http://www.hindawi.com
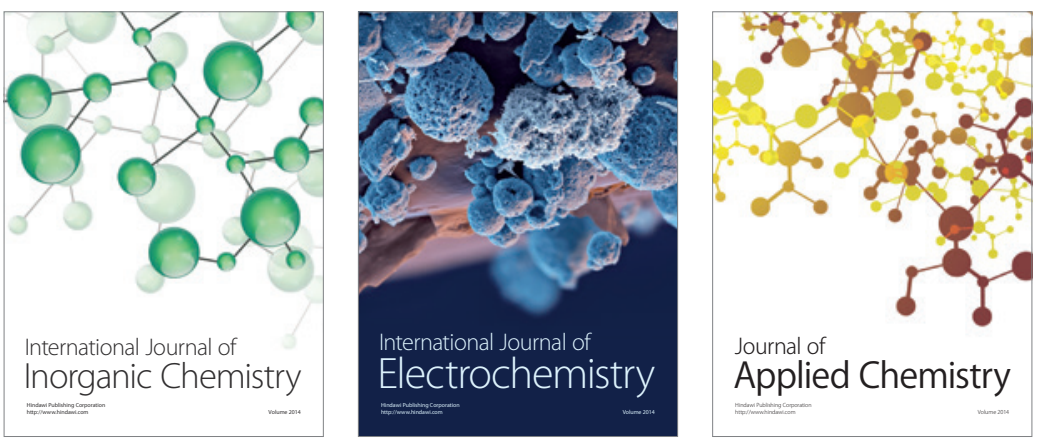

Journal of

Applied Chemistry
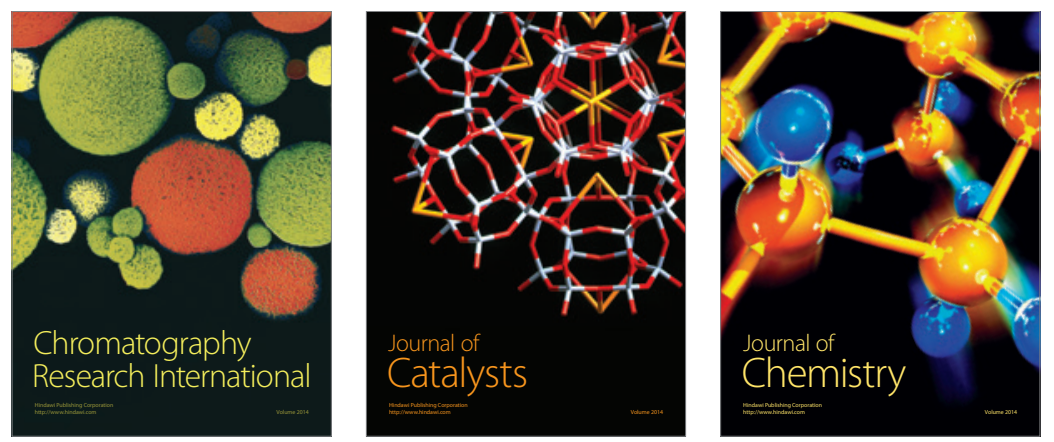
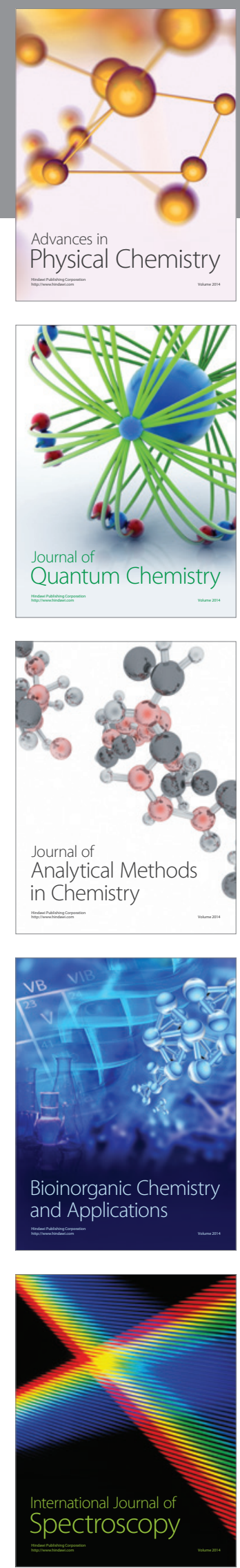